\title{
Glasdegib plus intensive/nonintensive chemotherapy in untreated acute myeloid leukemia: BRIGHT AML 1019 Phase III trials
}

\author{
Jorge E Cortes ${ }^{*} \ddagger, 1$, Hervé Dombret ${ }^{2}$, Akil Merchant ${ }^{3}$, Tetsuzo Tauchi ${ }^{4}$, Christine G \\ DiRienzo $^{5}$, Barbara Sleight ${ }^{5}$, Xiaoxi Zhang ${ }^{5}$, Eric P Leip ${ }^{5}$, Naveed Shaik ${ }^{5}$, Timothy Bell ${ }^{5}$, \\ Geoffrey Chan $^{5}$ \& Mikkael A Sekeres ${ }^{6}$ \\ ${ }^{1}$ Georgia Cancer Center, Augusta University, Augusta, GA 30912, USA. \\ ${ }^{2}$ Institut de Recherche Saint-Louis, Hôpital Saint-Louis, Assistance Publique-Hôpitaux de Paris Institut Universitaire d’Hematologie \\ Hopital Saint Louis, Paris, France \\ ${ }^{3}$ Department of Medicine, Keck School of Medicine, University of Southern California, Los Angeles, CA 90033, USA \\ ${ }^{4}$ Department of Hematology, Shin-Yurigaoka General Hospital, Kawasaki, Japan \\ ${ }^{5}$ Pfizer Oncology, Pfizer Inc., New York, NY 10017, USA \\ ${ }^{6}$ Leukemia Program, Cleveland Clinic, Cleveland, OH 44195, USA \\ *Author for correspondence: Tel.: +1 706-721-0570; jorge.cortes@augusta.edu \\ $\ddagger$ Affiliation when the manuscript was developed, submitted and accepted: Department of Leukemia, University of Texas, MD \\ Anderson Cancer Center, Houston, TX 77054, USA
}

Glasdegib, an oral Hedgehog pathway inhibitor, has been associated with significantly improved survival when combined with low-dose cytarabine in patients with untreated acute myeloid leukemia (AML) who were unsuitable for intensive chemotherapy, when compared with low-dose cytarabine alone. BRIGHT AML 1019 (NCT03416179) comprises two independently powered Phase III, randomized (1:1), double-blind global trials evaluating oral glasdegib $100 \mathrm{mg}$ once daily or placebo plus one of two standard chemotherapy regimens in adults with untreated AML. The intensive trial combines glasdegib/placebo with cytarabine and daunorubicin $(7+3)$, while the nonintensive trial combines glasdegib/placebo with azacitidine. The primary end point of both studies is overall survival. Secondary end points include response, time to and duration of response, event-free survival, safety, patient-reported outcomes and pharmacokinetics. Trial registration number: ClinicalTrials.gov identifier: NCT03416179

First draft submitted: 28 June 2019; Accepted for publication: 27 August 2019; Published online: 12 September 2019

Keywords: acute myeloid leukemia • glasdegib • Hedgehog signaling pathway • intensive chemotherapy • myelodysplastic syndrome $\bullet$ nonintensive chemotherapy $\bullet$ smoothened inhibitor

Acute myeloid leukemia (AML) is a genetically heterogeneous hematologic malignancy often characterized by multiple cytogenetic and molecular abnormalities at the time of diagnosis that influence the response to therapy, duration of response and overall survival (OS). AML is a rare disease [1-3]; existing treatment options for AML, such as a ' $7+3$ ' regimen of cytarabine plus an anthracycline or newly available first-line therapies (including a novel liposomal cytarabine-daunorubicin formulation for secondary AML, targeted therapies for different mutations and an antibody-drug conjugate), can induce complete remissions (CRs) in $50-80 \%$ of patients; however, refractory disease or relapse after an initial response occurs in $60-80 \%$ of patients [4-6]. Despite recent advances in therapy, new treatment options are still needed.

Leukemia stem cells (LSCs) can self-renew limitlessly, and can initiate AML development. Quiescent LSCs have been shown to be chemoresistant and responsible for disease persistence. Persistence of LSCs contributes to chemotherapy resistance and disease relapse. Chemotherapy may effectively debulk the disease in many instances, but an elusive goal of therapy for AML has been to eradicate these earliest leukemic progenitors (i.e., LSCs) to achieve a cure in more patients. Ideally, this would be achieved with less aggressive therapy and more tolerable therapeutic agents [7-9]. 
The Hedgehog (Hh) signaling pathway regulates cell differentiation and self-renewal in the developing embryo; it is typically silenced in adult tissues. Aberrant $\mathrm{Hh}$ signaling has been identified in a variety of human leukemias and LSCs [10]. Upregulation of Hh pathway components has been observed in vitro in AML cell lines that are resistant to chemotherapy, and pharmacological inhibition of the Hh pathway resulted in MDR-1 or P-gp expression [11]. Hh inhibitors act synergistically when combined with conventional chemotherapy in the treatment of hematologic cancers [12-14].

In normal tissue, the transmembrane protein smoothened (SMO) is essential for Hh pathway activation [13,15]. Studies using nonclinical models showed that SMO is involved in the maintenance of LSC dormancy acting via its effector GLI2, and is associated with resistance to chemotherapy and targeted therapy.

Glasdegib is a SMO inhibitor that has demonstrated potent and selective inhibition of Hh signaling in vitro, and significant antitumor efficacy in vivo [12,16-18]. SMO inhibition by glasdegib can cause LSCs to re-enter the cell cycle [16-20]. Hh signaling can be aberrantly activated in AML through epigenetic silencing of the pathway suppressor GLI3, and hypomethylating agents (HMAs) can reactivate GLI3 expression and modulate Hh signaling. The combination of hypomethylating agent and glasdegib has synergistic activity against AML [21].

Glasdegib demonstrated clinical activity in Phase I and Ib/II trials in patients with hematologic malignancies, including untreated AML or high-risk myelodysplastic syndromes (MDS) [22-28]. The Phase Ib/II trial (NCT01546038) of glasdegib in combination with ' $7+3$ ' in patients with AML or MDS showed evidence of clinical activity without additional safety concerns $[25,26]$. For adult patients with untreated AML who were considered unlikely to tolerate intensive chemotherapy, glasdegib plus low-dose cytarabine (LDAC) $(\mathrm{n}=78)$ improved OS compared with LDAC alone $(\mathrm{n}=38)$ (median 8.3 vs 4.3 months; hazard ratio [HR] 0.46; 80\% CI: 0.35-0.62, $95 \%$ CI: $0.30-0.72 ; \mathrm{p}=0.0002$ ). Rates of CR, CR with incomplete blood count recovery (CRi) and morphologic leukemia-free state (MLFS) were higher when patients were treated with glasdegib plus LDAC than with LDAC alone (26.9\%; 80\% CI: 20.5-33.4 vs 5.3\%; 80\% CI: 0.6-9.9). CR rate was also higher in the glasdegib plus LDAC group (17.9\%, $80 \%$ CI: $12.4-24.8$ vs $2.6 \%, 80 \%$ CI: $0.3-9.9)$ [27].

Based on these findings, glasdegib plus LDAC is approved in the USA for patients with untreated AML who are $\geq 75$ years or who have comorbidities that preclude intensive induction chemotherapy.

\section{Background \& rationale}

Glasdegib has been studied in $>300$ patients across multiple diseases [22-29]. The clinical benefit seen with the approximate doubling of OS in patients treated with glasdegib plus LDAC versus LDAC alone [27] constitutes the rationale for designing and executing this Phase III trial to further study glasdegib to understand its possible effects in combination with other standard-of-care chemotherapies for untreated adult AML.

\section{Glasdegib with daunorubicin \& cytarabine as intensive chemotherapy}

Standard ' $7+3$ ' induction chemotherapy with cytarabine and daunorubicin has been the mainstay of AML therapy for over 40 years. It induces remissions in $60 \%$ or more patients with untreated AML; however, despite postremission therapy including transplantation, a majority of patients relapse and die of AML [30-33].

Data from the open-label, single-arm Phase II glasdegib trial showed that among patients with untreated AML or MDS who were treated with glasdegib plus ' $7+3$ ' ( $\mathrm{n}=69), 46.4 \%$ (80\% CI: 38.7-54.1) achieved CR; the median OS was 14.9 months (80\% CI: $13.4-19.3)$ and the 12 -month survival probability was $66.6 \%$ (80\% CI: 58.5-73.4). Responses were observed across a variety of mutations (12 genes) [26]. Compared with historical data from risk-adjusted cohorts treated with cytarabine-based intensive regimens, OS appears better than expected following treatment with glasdegib plus ' $7+3$ ' [26].

\section{Glasdegib plus azacitidine as nonintensive chemotherapy}

Azacitidine has wide therapeutic application for patients with AML. It is approved in the European Union for patients with AML aged $\geq 65$ years who are not eligible for allogeneic hematopoietic stem cell transplantation (HSCT) [32,34], and regularly used in the USA for the same indication. The median OS in patients treated with azacitidine alone increased from 6.5 to 10.4 months compared with conventional care regimens, with a trend toward statistical significance [35]. In preclinical studies, SMO inhibitors (erismodegib [36] or glasdegib [18]) plus azacitidine showed synergistic activity. 


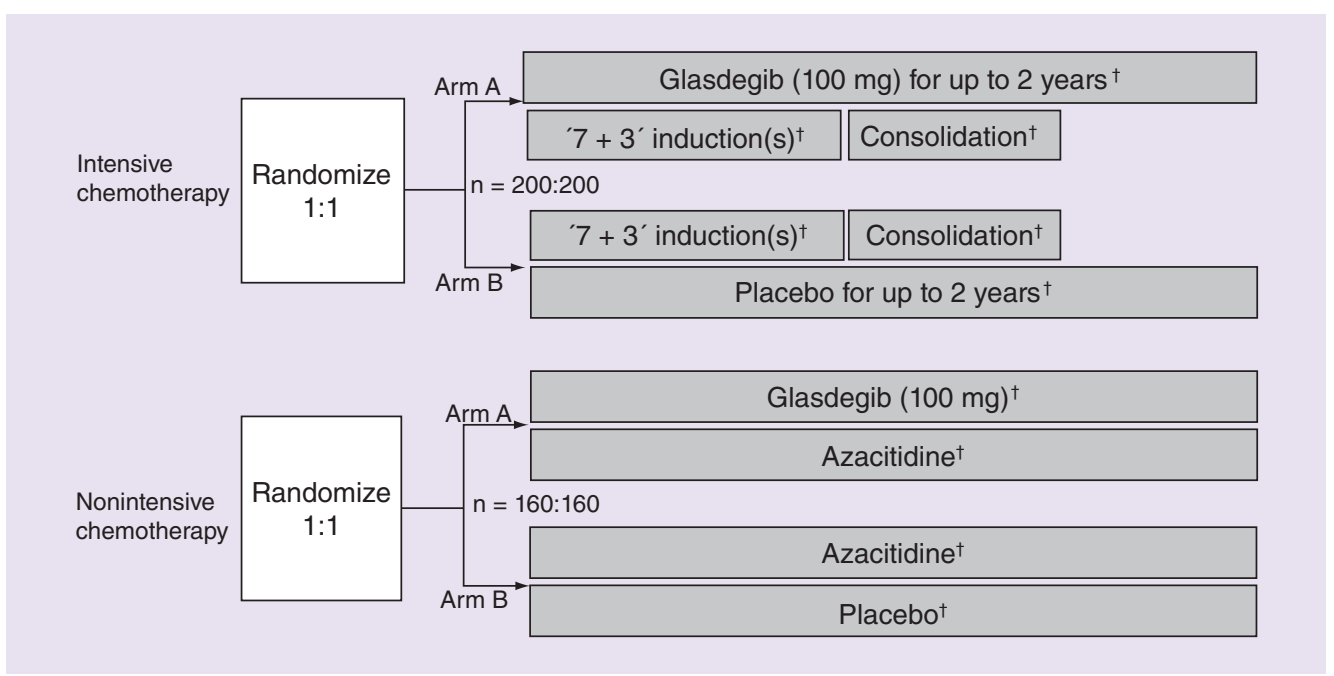

Figure 1. BRIGHT AML 1019 trial study design.

${ }^{\dagger}$ For dosing details, see Figures 2 \& 3 .

The Phase Ib trial of glasdegib plus azacitidine showed evidence of clinical activity with no evidence of drug-drug interaction; CRs were observed in 4 of the 12 patients, which appeared favorable in the context of the $15-17 \%$ CR rate seen with azacitidine alone [23].

\section{Glasdegib dosing \& schedule}

Based on the observed clinical benefit, safety and tolerability profile, modulation of Hh pathway and the likelihood and impact of concomitant cytochrome P450 3A4 inhibitor(s) on glasdegib exposure, the optimal starting dose of glasdegib in combination with chemotherapy was determined to be $100 \mathrm{mg}$ once daily (QD) given orally with or without food.

In the intensive therapy study, blinded therapy (glasdegib or placebo) is administered QD and continuously for up to 2 years postrandomization or until treatment failure, hematologic relapse, disease progression, toxicity, consent withdrawal, death or elimination of measurable/minimal residual disease (MRD) postconsolidation. In the nonintensive study, azacitidine and blinded therapy is scheduled to be administered for at least 6 cycles. Thereafter, therapy can continue indefinitely until disease progression, relapse, unacceptable toxicity, patient refusal or death.

In both the intensive and nonintensive studies, patients may receive HSCT. Eligible patients will stop study therapy 28 days prior to HSCT and may resume blinded therapy only 30-60 days post HSCT, when eligible. Single-agent blinded therapy will continue post HSCT until 2 years postrandomization, until achievement of MRD negative status, withdrawal of consent or disease progression, whichever comes first.

\section{Design}

\section{Study design}

BRIGHT AML 1019 includes two independent global studies in adult patients with untreated AML (Figure 1). This is an innovative, patient-centric design allowing treating physicians and patients to determine goals of care and enroll on either arm. In the intensive study, 400 patients will be randomized (1:1) to receive glasdegib or placebo, in combination with standard intensive chemotherapy. In the nonintensive study, 320 patients will be randomized $(1: 1)$ to receive glasdegib or placebo, in combination with azacitidine. Assignment to the intensive or nonintensive study will be decided by the investigator.

At randomization, patients will be stratified by the European LeukemiaNet (ELN) [37] genetic risk group (favorable vs intermediate vs adverse) and age. Patients will be randomized by a centralized interactive (web or voice) response technology system. Glasdegib or placebo treatment allocation will be blinded to the patient, the investigator and study site staff and the sponsor's study team. 


\section{Table 1. Objectives and end points.}

$\begin{array}{ll}\text { Primary objective: } & \text { Primary end point: } \\ \text { - To investigate whether glasdegib is superior to placebo in combination with } & \text { - OS } \\ \text { azacitidine (nonintensive study) or cytarabine and daunorubicin (intensive study) } & \end{array}$

azacitidine (nonintensive study) or cytarabine and daunorubicin (intensive study)

in prolonging $\mathrm{OS}$ in subjects with untreated $\mathrm{AML}$

Secondary objectives: Secondary end points:

- To compare fatigue score postbaseline as measured by MDASI-AML/MDS in both treatment arms

- To compare glasdegib vs placebo in combination with azacitidine (nonintensive

study) or ' $7+3$ ' (cytarabine and daunorubicin) in improving other clinical efficacy measures

- To estimate the duration of response in both treatment arms

- To estimate the time to response in both treatment arms in the nonintensive

study only

- To compare EFS in both treatment arms

- To compare PRO measurements in both treatment arms

- To evaluate the overall safety profile in both treatment arms

- To evaluate laboratory abnormalities in both treatment arms

- To characterize the PK of glasdegib

- To characterize treatment effects on the QTc interval

- Fatigue score measured by MDASI-AML/MDS questionnaire

- Rate of CR (including $C_{\text {MRD-neg }}$ as assessed by multiparametric flow cytometry), CRi as defined by the ELN recommendations (2017) [32], MLFS, PR and CRh for the nonintensive study only

- Duration of response (defined as $\mathrm{CR}$ [includes $\mathrm{CR}_{\mathrm{MRD} \text {-neg }}$ ]/CRi or $\mathrm{CR} / \mathrm{CRh}$ as appropriate)

- Time to response (CR [includes $\mathrm{CR}_{\mathrm{MRD} \text {-neg] }}$ /CRi or $\mathrm{CR} / \mathrm{CRh}$ as appropriate) in the nonintensive study only

- EFS

- PROs as measured by the MDASI-AML/MDS Module, EQ-5D-5L, PGIS and PGIC

- AEs as characterized by type, frequency, severity (as graded by NCI CTCAE v.4.03),

timing, seriousness and relationship to study therapy

- Laboratory abnormalities as characterized by type, frequency, severity (as graded

by $\mathrm{NCl}$ CTCAE v.4.03) and timing

- PK of glasdegib

- QTc interval

\section{Exploratory objectives:}

\section{Exploratory end point:}

- Bone marrow and blood biomarkers of response and/or resistance to glasdegib in combination with azacitidine or daunorubicin and cytarabine

- PROs for symptomatic AEs

- To collect banked biospecimens for exploratory research, unless prohibited by

local regulations or ethics committee decision

- Molecular, cellular and soluble markers in peripheral blood and/or bone marrow - As measured by a questionnaire containing two items from the PRO-CTCAE item library v.1.0 and an add-on item on muscle spasm

- Transfusion independence for the nonintensive study only

- Collection of banked bio specimens unless prohibited by local regulations or ethics committee decision

AE: Adverse event; AML: Acute myeloid leukemia; CR: Complete remission; $C R h$ : $C R$ with partial hematologic recovery; $C R i$ : $C R$ with incomplete blood count recovery; $C R$ MRD-neg: $C R$ with negative MRD; EFS: Event-free survival; ELN: European LeukemiaNet; EQ-5D-5L: EuroQoL 5-Dimension questionnaire 5-Level version; Hh pathway: Hedgehog pathway; MDASI-AML/MDS: M.D. Anderson symptom Inventory for AML/MDS; MDS: Myelodysplastic syndrome; MLFS: Morphologic leukemia-free state; MRD: Measurable/minimal residual disease; NCI CTCAE: National Cancer Institute Common Terminology Criteria for Adverse Event; OS: Overall survival; PGIC: Patient Global Impression of Change; PGIS: Patient Global Impression of Symptom; PK: Pharmacokinetic; PR: Partial remission; PRO: Patient-reported outcome; PRO-CTCAE: Patient-reported outcomes version of the Common Terminology Criteria for Adverse Event; QTC: Corrected QT interval.

\section{Study objectives}

The primary objective is to determine if glasdegib is superior to placebo in combination with cytarabine and daunorubicin (intensive trial) or azacitidine (nonintensive trial) in prolonging OS among patients with untreated AML. Table 1 shows the secondary and exploratory objectives and the relevant end points.

\section{Eligibility criteria}

Adult patients ( $\geq 18$ years, $\geq 20$ years in Japan) with untreated AML (using the World Health Organization 2016 classification [38], including AML evolved from MDS or antecedent hematologic disease or secondary AML) are eligible. Eligible patients must have adequate liver and renal function, in other words, serum aspartate aminotransferase and alanine aminotransferase $\leq 3 \times$ upper limit of normal, total serum bilirubin $\leq 2 \times$ upper limit of normal and estimated creatinine clearance $\geq 30 \mathrm{ml} / \mathrm{min}$; and QT interval corrected by the Fridericia Correction Formula $(\mathrm{QT} c \mathrm{cF}) \leq 470 \mathrm{~ms}$. Female patients of childbearing potential and male patients capable of fathering children must use highly effective contraception throughout the study and for 180 days after the last dose of study drug, whichever occurs later.

Key exclusion criteria include acute promyelocytic leukemia, AML with $B C R-A B L 1$ or $\mathrm{t}(9 ; 22)(\mathrm{q} 34 ; \mathrm{q} 11.2)$ as a sole abnormality, known active central nervous system leukemia, having another active malignancy on treatment, presence of a life-threatening or clinically significant uncontrolled systemic infection, or left ventricular ejection fraction $<50 \%$ (intensive trial only). Table 2 shows full eligibility criteria.

\section{Planned sample size}

For the intensive study stratified by ELN genetic risk and age ( $\leq 60$ vs $>60$ years), 400 patients with 267 deaths would provide $90 \%$ power to detect an improvement in OS with HR $=0.67$ (translated from a median OS of 21.0 vs 31.5 months), using a 1-sided log-rank test at a significance level of 0.025 and a 3-look group-sequential design. For adult patients ( $\geq 17$ years) with AML who received intensive therapy, the median OS was 23.7 months 


\section{Table 2. Eligibility criteria.}

\section{Inclusion criteria}

Patients must meet all of the following inclusion criteria to be eligible for enrollment into the intensive and nonintensive study (unless otherwise indicated):

1 Patients with untreated AML according to the WHO 2016 classification [38], including those with:

- AML arising from MDS or another AHD

- AML after previous cytotoxic therapy or radiation (secondary AML)

- FLT3+ AML, assuming the patient is not receiving and is not intended to receive FLT3 inhibitor therapy during study participation

$2 \quad \geq 18$ years of age ( $\geq 20$ years of age in Japan)

3 Adequate organ function as defined by the following:

- Serum AST and ALT $\leq 3 \times$ ULN, excluding patients with liver function abnormalities due to underlying malignancy

- Total serum bilirubin $\leq 2 \times$ ULN (except patients with documented Gilbert's syndrome)

- Estimated creatinine clearance $\geq 30 \mathrm{ml} / \mathrm{min}$ as calculated using the standard method for the institution

$4 \quad$ QTc interval $\leq 470$ ms using QTcF

- QTc criteria for eligibility does not apply to patients with a functioning pacemaker, whether the rhythm is paced or not

5 All anticancer treatments (unless specified) should be discontinued $\geq 2$ weeks from study entry, for example: targeted chemotherapy, radiotherapy, investigational anticancer agents, hormones or cytokines

- For control of rapidly progressing leukemia, hydroxyurea and/or leukapheresis may be used before and for up to 1 week after the first dose of glasdegib. Continuation or resumption of hydroxyurea or leukapheresis after that time period must be approved by the study sponsor

6 Serum or urine pregnancy test (for female patients of childbearing potential) with a minimum sensitivity of 25 IU/I or equivalent units of hCG-negative at screening

$7 \quad$ Male and female patients of childbearing potential and at risk for pregnancy must agree to use at least one highly effective method of contraception throughout the study and for 180 days after the last dose of azacitidine, cytarabine or daunorubicin; and the last dose of glasdegib or placebo, whichever occurs later

8 Female patients of nonchildbearing potential must meet at least 1 of the following criteria:

- Have undergone a documented hysterectomy and/or bilateral oophorectomy;

- Have medically confirmed ovarian failure; or

- Have achieved postmenopausal status, defined as follows: cessation of regular menses for at least 12 consecutive months with no alternative pathological or physiological cause; status may be confirmed by having a serum FSH level confirming the postmenopausal state All other female patients (including female patients with tubal ligations) are considered to be of childbearing potential

9 Consent to a saliva sample collection for a germline comparator, unless prohibited by local regulations or ethics committee decision

10 Evidence of a personally signed and dated informed consent document indicating that the patient has been informed of all pertinent aspects of the study

11 Patients who are willing and able to comply with the study-scheduled visits, treatment plans, laboratory tests and other procedures (including bone marrow assessments)

\section{Exclusion criteria}

Patients with any of the following characteristics/conditions will not be included in the study:

1 APL and APL with PML-RARA (WHO 2016 classification) [38]. This disease is associated with $\mathrm{t}(15 ; 17)$

$2 \quad$ AML with $B C R-A B L 1$ or $\mathrm{t}(9 ; 22)(\mathrm{q} 34 ; \mathrm{q} 11.2)$ as a sole abnormality - Complex genetics may include $t(9 ; 22)$ cytogenetic translocation

$3 \quad$ Patients with known active CNS leukemia

4 Participation in other clinical studies involving other investigational drug(s) (Phases I-IV) within 4 weeks prior to study entry and/or during study participation

5 Patients known to be refractory to platelet or packed red cell transfusions per institutional guidelines, or a patient who refuses blood product support

6 Patients with another active malignancy on treatment with the exception of basal cell carcinoma, nonmelanoma skin cancer, cervical carcinoma in situ. Other prior or concurrent malignancies will be considered on a case-by-case basis

7 Any one of the following ongoing or in the previous 6 months: myocardial infarction, congenital long QT syndrome, torsades de pointes, symptomatic arrhythmias (including sustained ventricular tachyarrhythmia), right or left bundle branch block and bifascicular block, unstable angina, coronary/peripheral artery bypass graft, symptomatic CHF (CHF New York Heart Association Class III or IV), cerebrovascular accident, transient ischemic attack or symptomatic pulmonary embolism; as well as bradycardia defined as $<50 \mathrm{bpm}$

8 Patients with an active, life-threatening or clinically significant uncontrolled systemic infection not related to AML

9 Patients with LVEF $<50 \%$ are excluded from the intensive chemotherapy study only

10 Cumulative anthracycline dose equivalent of $\geq 550 \mathrm{mg} / \mathrm{m}^{2}$ of daunorubicin for the intensive chemotherapy study only

11 Known malabsorption syndrome or other condition that may significantly impair absorption of study medication in the investigator's judgment (e.g., gastrectomy, lap band and Crohn's disease) and inability or unwillingness to swallow tablets or capsules

12 Current use or anticipated requirement for drugs that are known strong CYP3A4/5 inducers

13 Concurrent administration of herbal preparations

14 Major surgery or radiation within 4 weeks of starting study treatment

AHD: Antecedent hematologic disease; ALT: Alanine aminotransferase; AML: Acute myeloid leukemia; APL: Acute promyelocytic leukemia; AST: Aspartate aminotransferase; CHF: Congestive heart failure; CNS: Central nervous system; CYP3A4/5: Cytochrome P450 3A4/5; FSH: Follicle-stimulating hormone; hCG: Human chorionic gonadotropin LVEF: Left ventricular ejection fraction; MDS: Myelodysplastic syndrome; PML-RARA: Promyelocytic leukemia/retinoic acid receptor alpha; QTC: Corrected QT interval; QTCF: Fridericia formula for QT interval correction; ULN: Upper limit of normal. 


\section{Table 2. Eligibility criteria (cont.).}

\begin{tabular}{|c|c|}
\hline \multicolumn{2}{|r|}{$\begin{array}{l}\text { Exclusion criteria } \\
\text { Patients with any of the following characteristics/conditions will not be included in the study: }\end{array}$} \\
\hline 15 & $\begin{array}{l}\text { Documented or suspected hypersensitivity to any one of the following: } \\
\text { - For patients assigned to intensive chemotherapy, documented or suspected hypersensitivity to cytarabine (not including drug fever or } \\
\text { exanthema, including known cerebellar side effects) or daunorubicin } \\
\text { - For patients assigned to nonintensive chemotherapy, documented or suspected hypersensitivity to azacitidine or mannitol }\end{array}$ \\
\hline 16 & Known active drug or alcohol abuse \\
\hline 17 & $\begin{array}{l}\text { Other acute or chronic medical or psychiatric condition including recent (within the past year) or active suicidal ideation or behavior or laboratory } \\
\text { abnormality that may increase the risk associated with study participation or investigational product administration or may interfere with the } \\
\text { interpretation of study results and, in the judgment of the investigator, would make the subject inappropriate for entry into this study }\end{array}$ \\
\hline 18 & Pregnant or breastfeeding female patients \\
\hline 19 & Known recent or active suicidal ideation or behavior \\
\hline 20 & $\begin{array}{l}\text { Investigator site staff members directly involved in the conduct of the study and their family members, site staff members otherwise supervised by the } \\
\text { investigator, or patients who are employees of the sponsor, including their family members, directly involved in the conduct of the study }\end{array}$ \\
\hline & $\begin{array}{l}\text { ecedent hematologic disease; ALT: Alanine aminotransferase; AML: Acute myeloid leukemia; APL: Acute promyelocytic leukemia; AST: Aspartate aminotransferase; } \\
\text { gestive heart failure; CNS: Central nervous system; CYP3A4/5: Cytochrome P450 3A4/5; FSH: Follicle-stimulating hormone; hCG: Human chorionic gonadotropin; } \\
\text { ventricular ejection fraction; MDS: Myelodysplastic syndrome; PML-RARA: Promyelocytic leukemia/retinoic acid receptor alpha; QTC: Corrected QT interval; QTCF: } \\
\text { formula for QT interval correction; ULN: Upper limit of normal. }\end{array}$ \\
\hline
\end{tabular}

for those $\leq 60$ years and 15 months for those $>60$ years [39,40]; therefore, a composite median OS of 21 months was assumed.

For the nonintensive trial, patients will be stratified by ELN genetic risk and age ( $<75$ vs $\geq 75$ years); 320 patients with 220 deaths would provide $90 \%$ power to detect an improvement in OS with $\mathrm{HR}=0.64$ (translated from a median OS of 10.4 months [patients $\geq 65$ years with untreated AML and $>30 \%$ bone marrow blasts] [35] vs 16.2 months) using a 1-sided log-rank test at a significance level of 0.025 and a 2-look group-sequential design.

\section{Planned study period}

Assuming that enrollment increases steadily for 10 months to reach 18 patients per month, then plateaus at that rate, the estimated time is 28 and 23 months to enroll 400 and 320 patients in the intensive and nonintensive chemotherapy studies, respectively. The estimated number of death events will accrue within 58 and 37 months after the first patient was randomized into each study; however, the actual time will be affected by actual patient enrollment and dropout rates.

\section{Dose \& therapy}

Patients randomized in the intensive study (Figure 2) will receive glasdegib $100 \mathrm{mg}$ QD or placebo (blinded therapy) on Day 1 and continue for up to 2 years or until disease progression, toxicity or MRD-negative postconsolidation and/or HSCT. ' $7+3$ ' induction will consist of intravenous (IV) cytarabine $100 \mathrm{mg} / \mathrm{m}^{2}$ for 7 days + daunorubicin $60 \mathrm{mg} / \mathrm{m}^{2}$ for 3 days; if a second induction is indicated, ' $7+3$ ' or ' $5+2$ ' will be used per physician's choice. Consolidation consists of single-agent IV cytarabine $1 \mathrm{~g} / \mathrm{m}^{2}$ (patient $\geq 60$ years) or $3 \mathrm{~g} / \mathrm{m}^{2}$ (patient $<60$ years) over $3 \mathrm{~h}$ twice daily on Days 1, 3 and 5 every 28 days for up to 4 cycles (alternative schedules may be used). Patients who proceed to HSCT will stop blinded therapy at least 28 days prior to HSCT, and may resume blinded therapy 30-60 days post-HSCT assuming absolute neutrophil count (ANC) engraftment $\left(\geq 0.5 \times 10^{9} / 1\right.$ for 3 consecutive days without growth factor support), no ongoing $\geq$ grade 2 graft-versus-host disease (GvHD) and no ongoing serious adverse events (SAEs) that prevent resumption of glasdegib/placebo in the judgment of the investigator.

Patients randomized in the nonintensive study (Figure 3) will receive blinded therapy continuously in combination with azacitidine $\left(75 \mathrm{mg} / \mathrm{m}^{2}\right)$ given subcutaneously or intravenously for 7 days in 28-day cycles. Treatment will continue for at least 6 cycles, or until unacceptable toxicity, disease progression, patient refusal or death, whichever occurs first.

In both the intensive and nonintensive studies, patients who proceed to HSCT will interrupt study therapy 28 days prior to HSCT. Single-agent blinded therapy may be resumed 30-60 days post-HSCT once there is ANC engraftment, no $\geq$ grade $2 \mathrm{GvHD}$ and no ongoing serious adverse events (SAEs) that prevent resuming blinded therapy in the judgment of the investigator. Blinded therapy may continue for up to 2 years postrandomization, until MRD negativity is documented postconsolidation and/or HSCT on two consecutive evaluations, withdrawal, 


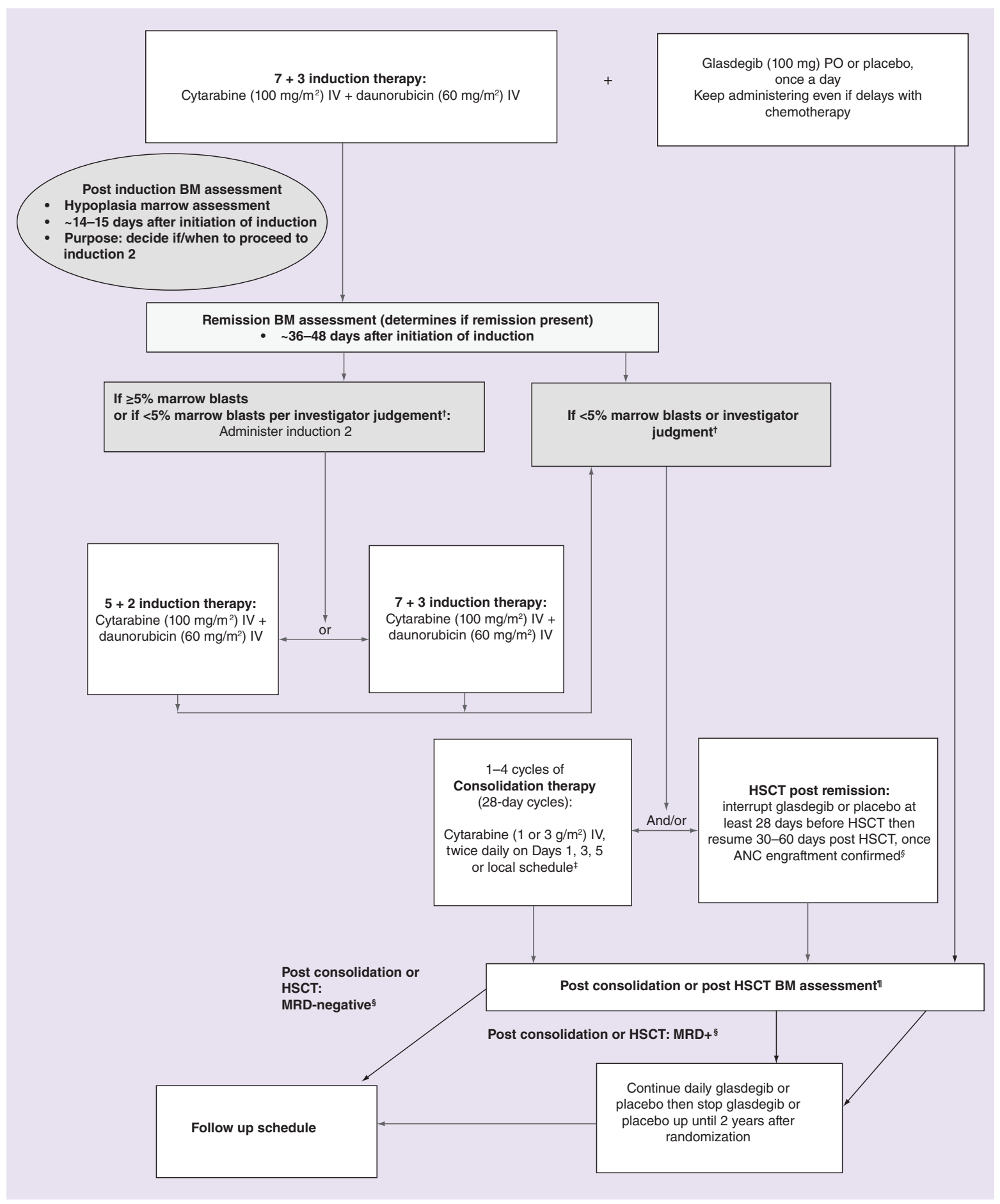

Figure 2. Intensive chemotherapy treatment algorithm.

†If $<5 \%$ Bone marrow (BM) blasts, the investigator may choose to start a second induction therapy or proceed to consolidation therapy with single-agent cytarabine and/or postremission HSCT.

$\ddagger$ Consolidation with cytarabine $3 \mathrm{~g} / \mathrm{m}^{2} \mathrm{IV}$ for adults $<60$ years and $1 \mathrm{~g} / \mathrm{m}^{2}$ for adults $\geq 60$ years over $3 \mathrm{~h}, \mathrm{BID}$, on Days 1,3 and 5 , every 28 days for up to 4 cycles. Alternative single-agent cytarabine consolidation schedules may be used per local prescribing information. Resume glasdegib or placebo 30-60 days post day of stem cell infusion, once there is confirmed ANC engraftment ( $\left.\geq 0.5 \times 10^{9} / \mathrm{l}\right)$ for 3 consecutive days with no growth factor support, no $\geq$ grade 2 ongoing GvHD and no ongoing SAEs that prevent resuming glasdegib or placebo in the judgment of the investigator. Following post consolidation or post HSCT, modify treatment based on two matching and consecutive MRD results (by central laboratory) when they are available. MRD test results are not required prior to continuing glasdegib or placebo, however, modify treatment when results are available.

I Post consolidation BM assessment should be conducted after chemotherapy-based consolidation period and before any subsequent treatment. For patients proceeding to HSCT, post-HSCT BM assessment will be conducted approximately 3 months after HSCT. This post-HSCT BM assessment may be later than the resumption of glasdegib or placebo 30-60 days post HSCT.

ANC: Absolute neutrophil count; BID: Twice daily; BM: Bone marrow; GvHD: Graft-versus-host disease; HSCT: Hematopoietic stem cell transplantation; IV: Intravenous; MRD: Measurable/minimal residual disease; PO: By mouth; SAE: Serious adverse event. 


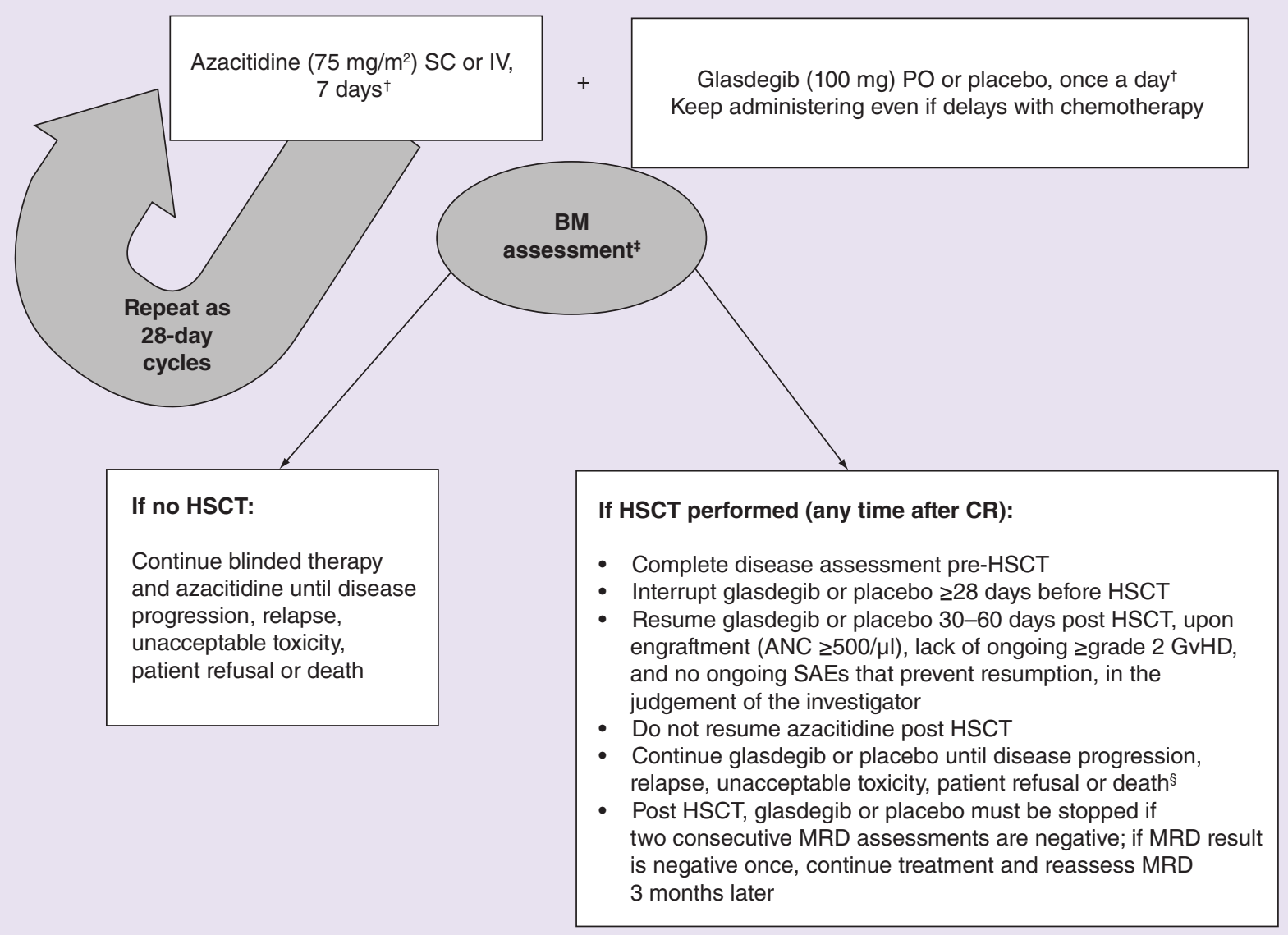

Figure 3. Nonintensive chemotherapy treatment algorithm.

†Details for azacitidine administration with glasdegib or placebo are described in study protocol.

$\ddagger$ A Bone marrow (BM) assessment is required when omplete remission is suspected or after end of Cycle 6, whichever is earlier. BM aspirate is required, and biopsy is preferred. Following Cycle 6, BM assessments will occur when there is an intention for patient to receive HSCT, or when progressive disease is suspected.

$\S$ Post-HSCT, glasdegib or placebo is administered up to 2 years after randomization.

ANC: Absolute neutrophil count; BM: Bone marrow; CR: Complete remission; GvHD: Graft-versus-host disease; HSCT: Hematopoietic stem cell transplantation; IV: Intravenous; MRD: Measurable/minimal residual disease; PO: By mouth; SAE: Serious adverse event; SC:

Subcutaneous.

disease progression, relapse, unacceptable toxicity or death. Glasdegib or placebo may continue regardless of chemotherapy dose modifications/delays.

\section{Study procedures}

Screening may occur up to 28 days. All assessments on Day 1 of study therapy will be collected predose, unless documented otherwise. Patients may withdraw from the study at any time by their own request, or as advised by the investigator. Follow-up for survival is up to 5 years from the first dose of the last patient enrolled in the study, or until death, or consent withdrawal.

\section{Efficacy end points \& assessments}

Efficacy end points include OS, event-free survival (EFS), rates of response (CR, CR with negative MRD $\left[\mathrm{CR}_{\mathrm{MRD}-\mathrm{neg}}\right]$, CRi, CR with partial hematologic recovery [CRh, nonintensive only], MLFS and partial response $[\mathrm{PR}])$ and duration of and time to response. OS is the primary end point of both studies, defined as time from randomization to death due to any cause.

EFS is defined as time from randomization to treatment failure, relapse from CR or CRh (nonintensive only) or death from any cause. Treatment failure is failure to achieve CR during the induction cycle (including the re- 
Table 3. Glasdegib or placebo dose modifications for nonhematologic toxicities excluding QTc prolongation, muscle spasms and myalgia.

\begin{tabular}{|c|c|}
\hline Toxicity & Glasdegib or placebo \\
\hline $\begin{array}{l}\geq \text { Grade } 3 \text { toxicity nausea, vomiting and/or diarrhea persist at } \geq \text { grade } 3 \text { despite } \\
\text { maximal appropriate medical therapy }\end{array}$ & $\begin{array}{l}\text { Hold study drug until toxicity has recovered to baseline or } \leq \text { grade } 1 \\
\text { - First episode: decrease by } 1 \text { dose level } \\
\text { - Second episode: decrease by } 1 \text { dose level } \\
\text { - Third episode: permanently discontinue } \\
\text { Dose escalations are not allowed following glasdegib or placebo dose reductions }\end{array}$ \\
\hline Potential DILI ${ }^{\dagger}$ & $\begin{array}{l}\text { Interrupt study drug dosing } \\
\text { If an alternative cause is found, restarting of glasdegib or placebo at the same dose } \\
\text { may be considered }\end{array}$ \\
\hline Confirmed DILI ${ }^{\dagger}$ & Permanently discontinue study drug \\
\hline \multicolumn{2}{|c|}{$\begin{array}{l}\text { Toxicity is graded by the National Cancer Institute Common Terminology Criteria for Adverse Events (NCI CTCAE) version } 4.03 \text {. } \\
\text { †DILI is assessed per Hy's law criteria. Patients with normal baseline AST, ALT and TBili values who have AST or ALT values }>3 \times \text { ULN, TBili value }>2 \times \text { ULN with no evidence of hemolysis, } \\
\text { and alkaline phosphatase value }<2 \times \text { ULN or not available are evaluated further as potential DILI cases to definitively determine the etiology of the abnormal laboratory values. Patients } \\
\text { with baseline AST or ALT values above the ULN are evaluated further as potential DILI cases if they have AST or ALT values more than two-times the baseline values and }>3 \times \text { ULN or } \\
>8 \times \text { ULN, whichever is smaller. Patients with baseline TBili above the normal range are evaluated further as potential DILI cases if their TBili level increases from baseline value by an } \\
\text { amount of at least } 1 \times \text { ULN or if the value reaches }>3 \times \text { ULN, whichever is smaller. } \\
\text { ALT: Alanine aminotransferase; AST: Aspartate aminotransferase; DILI: Drug-induced liver injury; QTC: Corrected QT interval; TBili: Total bilirubin; ULN: Upper limit of normal. }\end{array}$} \\
\hline
\end{tabular}

induction cycle if applicable) for intensive therapy, and failure to achieve CR or CRh following up to 6 cycles of study treatment for nonintensive therapy.

Disease response is evaluated using the 2017 ELN recommendations for diagnosis and management of AML in adults [32], including CR, with MRD status as assessed centrally by multiparametric flow cytometry; CRi; MLFS; PR; and CRh for the nonintensive study only. CRh is defined as CR but with ANC $>0.5 \times 10^{9} / 1$ and platelets $>50 \times 10^{9} / 1$.

Duration of response (defined as time from CR [includes $\mathrm{CR}_{\mathrm{MRD}-\mathrm{neg}}$ ]/CRi or CR/CRh as appropriate to disease progression, or relapse after $\mathrm{CRi} / \mathrm{CRh}$ or better, or death due to any cause) will be assessed. Time to response (defined as time from randomization to $\mathrm{CR}$ [includes $\mathrm{CR}_{\mathrm{MRD} \text {-neg }}$ / CRi or CR/CRh as appropriate for responders only) will be assessed in the nonintensive study only.

Genetic risk will be classified according to the 2017 ELN guideline [32]. A bone marrow aspirate sample will be required at screening for all patients, and a bone marrow biopsy preferred. No bone marrow assessments (BMA) will be necessary if nonresponse or progressive disease (PD) can be confirmed from peripheral blood evaluation or radiological/clinical assessment. For the intensive study, when applicable, BMA will be performed shortly after completion of the first induction chemotherapy (to determine if/when the second induction should be started), at the end of induction upon at least partial hematologic recovery, at the end of consolidation period and pre- and post-HSCT. A disease assessment will be performed once yearly for patients in remission and whenever relapse is suspected as appropriate. For the nonintensive study, BMA will be performed when CR is suspected or after cycle 6 (whichever is earlier), pre-HSCT, once yearly for patients in remission and when PD is suspected.

All bone marrow samples will be evaluated by a local laboratory and assessed by the investigator for response. In addition, bone marrow aliquots will be shipped to a central laboratory for MRD analysis and molecular profiling.

\section{Safety assessments}

Safety assessments will include collection of AEs, SAEs, vital signs, complete physical examination including performance status, triplicate 12-lead electrocardiogram (ECG), routine laboratory assessments, transfusions, HSCTrelated information and verification of concurrent medications, and other interventions. Severity of AEs will be graded by the National Cancer Institute Common Terminology Criteria for Adverse Events (CTCAE, version 4.03). Acute and chronic GvHD will be assessed and graded using standard criteria [41-43].

Dose of blinded therapy will be modified (dose reduction, treatment interruption or discontinuation) if patients experience treatment-related grade 3-4 nonhematological toxicities (nausea, vomiting and/or diarrhea persist at $\geq$ grade 3 despite maximal appropriate medical therapy, potential and confirmed drug-induced liver injury [Hy's Law]); grade 2-3 muscle spasms or myalgia; or grade 2-4 mean QTcF prolongation (Tables 3-5). 
Table 4. Glasdegib or placebo dose modifications and other management procedures for mean QTcF prolongation.

\begin{tabular}{|c|c|c|c|c|c|}
\hline \multirow[t]{2}{*}{ Category } & \multirow[t]{2}{*}{ Action } & \multicolumn{4}{|c|}{ Grade of QTc prolongation } \\
\hline & & 1 & 2 & 3 & 4 \\
\hline \multirow[t]{2}{*}{ General management } & Assess electrolyte levels and supplement as clinically indicated & & $\mathrm{X}$ & $\mathrm{x}$ & $\mathrm{x}$ \\
\hline & Review and adjust concomitant medications with known QTc interval-prolonging effects & & $\mathrm{x}$ & $\mathrm{x}$ & $\mathrm{x}$ \\
\hline ECG monitoring & Monitor ECGs at least weekly for 2 weeks following resolution of mQTcF prolongation to $\leq 480 \mathrm{~ms}$ & & $\mathrm{x}$ & $\mathrm{x}$ & $\mathrm{x}$ \\
\hline \multirow[t]{3}{*}{ Initial glasdegib action } & Discontinue and do not rechallenge & & & & $\mathrm{x}$ \\
\hline & Interrupt treatment & & & $\mathrm{x}$ & \\
\hline & Continue treatment at same dose & $\mathrm{x}$ & $\mathrm{x}$ & & \\
\hline \multirow[t]{2}{*}{$\begin{array}{l}\text { Resume glasdegib } \\
\text { dosing }\end{array}$} & $\begin{array}{l}\text { If no prior glasdegib dose interruption related to QTcF prolongation has occurred, resume at a } \\
\text { reduced dose of } 75 \mathrm{mg} \text { once daily when mQTcF interval returns to within } 30 \mathrm{~ms} \text { of baseline or } \\
\leq 480 \mathrm{~ms}\end{array}$ & & & $\mathrm{x}$ & \\
\hline & $\begin{array}{l}\text { If one prior glasdegib dose interruption related to QTcF prolongation has occurred, resume at a } \\
\text { reduced dose of } 50 \mathrm{mg} \text { once daily when mQTcF interval returns to within } 30 \mathrm{~ms} \text { of baseline or } \\
\leq 480 \mathrm{~ms}\end{array}$ & & & $\mathrm{x}$ & \\
\hline $\begin{array}{l}\text { Discontinue glasdegib } \\
\text { permanently }\end{array}$ & If two prior glasdegib dose interruptions related to QTcF prolongation have occurred & & & $\mathrm{x}$ & \\
\hline \multicolumn{6}{|c|}{$\begin{array}{l}\text { The severity of QTc prolongation is assessed by calculating a mean QT of three consecutive ECGs performed } \sim 2 \mathrm{~min} \text { (but no longer than } 5 \mathrm{~min} \text { ) apart using the QTcF. When QTC } \\
\text { prolongation is } 450-480 \mathrm{~ms} \text {, the severity is grade 1; QTc at } 481-500 \mathrm{~ms} \text { is grade 2; and QTc } \geq 501 \mathrm{~ms} \text { for at least two separate ECGs is grade } 3 \text {. If QTc } \geq 501 \mathrm{~ms} \text { or }>60 \mathrm{~ms} \\
\text { change from baseline and torsade de pointes or polymorphic ventricular tachycardia or signs/symptoms of serious arrhythmia, the severity is grade } 4 \text {. } \\
\text { ECG: Electrocardiogram; mQTCF: Dose modifications for mean QTCF; QTc: Corrected QT; QTCF: Fridericia formula for QT interval correction. }\end{array}$} \\
\hline
\end{tabular}

\begin{tabular}{|c|c|c|c|}
\hline \multirow[t]{2}{*}{ Glasdegib or placebo dose and other management procedures } & \multicolumn{3}{|c|}{ Muscle spasms and myalgia } \\
\hline & Grade 1 & Grade 2 & Grade 3 \\
\hline Continue at same dose level & $\mathrm{x}$ & $\mathrm{x}$ & \\
\hline Hold dose & & & $\mathrm{x}$ \\
\hline${\text { Administer oral rehydration solutions containing electrolytes }{ }^{\dagger}}$ & $\mathrm{x}$ & $\mathrm{x}$ & $\mathrm{x}$ \\
\hline Consider other appropriate interventions (e.g., antispasmodics) as per institutional guidelines & $\mathrm{x}$ & $x$ & $\mathrm{x}$ \\
\hline Evaluate levels of $\mathrm{CK}$, Vit B6, Vit D, ferritin, transferrin and electrolytes $(\mathrm{Na}, \mathrm{K}, \mathrm{Mg}, \mathrm{Ca} \text { and } \mathrm{P})^{\ddagger}$ & $x$ & $x$ & $\mathrm{x}$ \\
\hline If CK is elevated, evaluate urine and serum myoglobin, CK isoenzymes and plasma creatinine & $x$ & $\mathrm{x}$ & $x$ \\
\hline $\begin{array}{l}\text { If event persists, hold dose until resolution to grade } \leq 1 \text {. Upon resolution, restart at prior dose, or for prolonged } \\
\text { muscle spasms, consider reducing dose by one dose level }\end{array}$ & & $\mathrm{x}$ & \\
\hline $\begin{array}{l}\text { Upon resolution to grade } \leq 1 \text {, restart study treatment at next lower dose level. If the event does not resolve within } \\
3 \text { weeks to grade } \leq 1 \text {, at the discretion of the investigator the dose may be restarted at the next lower dose level } \\
\text { or the patient may be permanently discontinued from study treatment }\end{array}$ & & & $\mathrm{x}$ \\
\hline \multicolumn{4}{|c|}{$\begin{array}{l}\text { In the event of alopecia or dysgeusia, investigator discretion should be applied with respect to dose interruption and/or dose reduction of glasdegib/placebo as preliminary } \\
\text { analysis of available clinical data suggests that these events are not dose dependent. } \\
\text { †Electrolyte replacement drinks should include } \mathrm{Na}, \mathrm{K}, \mathrm{Mg}, \mathrm{Ca} \text { and P. Consideration should be given to ensuring adequate hydration prior to bedtime, and whenever fluid intake } \\
\text { is decreased for a prolonged duration. } \\
\text { ¥Samples for laboratory examinations may be taken as unscheduled assessments between protocol visits. } \\
\text { Ca: Calcium; CK: Creatinine kinase; K: Potassium; Mg: Magnesium; Na: Sodium; P: Phosphorus; Vit: Vitamin. }\end{array}$} \\
\hline
\end{tabular}

\section{Biomarker assessments}

Biomarker assessments on all enrolled patients will be performed at a central laboratory. Bone marrow aspirate will be used for MRD assessment and molecular profiling, peripheral blood for soluble factor assessments and saliva sample is used as a germline comparator.

\section{Pharmacokinetics of glasdegib}

The pharmacokinetics (PK) of glasdegib will be analyzed using a validated method. A sparse PK sampling approach will be utilized in this double-blind study, with limited PK samples collected during induction/consolidation (intensive study) and Cycles 1-3 (nonintensive study). PK samples obtained within 10\% of the nominal time (e.g., within 6 min of a 60-min sample) will be considered protocol-compliant. The predose PK sample should be collected within $30 \mathrm{~min}$ before administration of glasdegib. 


\section{Pharmacogenomics}

Banked biospecimens (whole blood samples) will be collected from patients for exploratory research relating to the drug and AML. Biospecimens will be used only for the purposes described in the protocol and informed consent document; any other uses require additional ethical approval.

\section{Patient-reported outcomes}

Patient-reported outcomes (PRO) will be measured using the MD Anderson Symptom Inventory AML/MDS Module (MDASI-AML/MDS), EuroQol 5-Dimension Questionnaire 5-Level version (EQ-5D-5L), Patient Global Impression of Symptoms (PGIS) and Patient Global Impression of Change (PGIC). In addition, a PRO version of the CTCAE (PRO-CTCAE) will be used. Fatigue (tiredness) score measured by the MDASI-AML/MDS questionnaire is a key secondary efficacy end point. PGIC is completed at all postbaseline time points, without an initial baseline assessment.

\section{Data analysis/statistical methods}

Analysis population

The full analysis set will include all randomized patients. Patients will be classified by treatment assigned at randomization. The safety analysis set will include all patients who receive $\geq 1$ dose of study drug. Patients will be classified by treatment assigned at randomization unless the incorrect treatment $(s)$ was/were received throughout the dosing period, in which case patients will be classified according to the first study treatment received.

PK analysis will be carried out on the PK concentration analysis set, defined as all patients who are treated and who have $\geq 1$ value of analyte concentration of glasdegib available, and the PK parameter analysis set, defined as all patients who are treated and who have $\geq 1$ of the PK parameters of interest.

The biomarker analysis set is defined as all patients who receive $\geq 1$ dose of any study treatment and have $\geq 1$ valid baseline biomarker assessment.

\section{End point analysis}

Efficacy will be based on all randomized patients, while analysis of safety and other end points will be carried out in the relevant patient sets as defined above.

The primary analysis of OS will be performed based on the full analysis set. A one-sided stratified log-rank test will be used with the overall significance level preserved at one-sided $\leq 0.025$. For the intensive chemotherapy population, this will happen when approximately 267 death events have occurred; the test is stratified by ELN genetic risk and age $\leq 60$ versus $>60$ years. For the nonintensive chemotherapy population, this will happen when approximately 220 death events have occurred; the test is stratified by ELN genetic risk and age $<75$ versus $\geq 75$ years. OS time associated with each treatment arm will be summarized using the Kaplan-Meier method and, where appropriate, CI for the 25th, 50th (median) and 75th percentiles will be reported (using the Brookmeyer and Crowley method and $\log -\log$ transformation) [44,45]. Should over-stratification prevent model convergence or if a large imbalance exists in the distribution (e.g., $\geq 90 \%$ patients from one strata level), the intermediate and poor genetic risk groups may be pooled in the analysis. The Cox proportional hazards model will be fitted to compute the treatment HR and the corresponding 95\% CI.

The proportion of patients achieving each disease-specific efficacy end point (CR, CRh, CRi, MLFS and PR) will be estimated with two-sided 95\% CI, respectively (using normal approximation). The proportion of patients achieving each end point will be compared between the treatment arms using one-sided Cochran Mantel Haenszel stratified test and unstratified $\chi^{2}$ test. Duration of response will be analyzed using the Kaplan-Meier method; median and corresponding two-sided $95 \%$ CI will be provided. Time to response for responders only will be summarized descriptively (median, minimum and maximum).

EFS will be analyzed similarly to OS. Descriptive statistics including means, medians, standard deviations and 95\% CIs will be provided for PROs. Statistical comparison of the two treatment groups for continuous PRO scores will be based on a longitudinal repeated measures mixed effects model using baseline as a covariate.

The key secondary PRO end point based on the fatigue (tiredness) item from the MDASI-AML/MDS questionnaire will be analyzed for proportion of responders at weeks 8 and 12 for intensive and nonintensive chemotherapy patients, respectively. The proportion of responders will be estimated with two-sided 95\% CI (using normal approximation). The proportion of responders and two-sided $95 \% \mathrm{CI}$ (using exact method) for each stratum will also be provided. A hierarchical testing procedure will be followed, namely, a formal test of proportion of responders 
(using one-sided Cochran Mantel Haenszel test, stratified by ELN genetic risk and age) will only be conducted in one population if the primary OS end point is met in that population (intensive or nonintensive treatment). The risk ratio and two-sided $95 \% \mathrm{CI}$ will also be summarized. Responder definition will be determined via anchor-based methods using PGIS (primary) and PGIC (supportive), which will be supplemented with cumulative distribution functions and probability density functions (PDF). Cumulative distribution function and PDF will be created using individual change from baseline for the fatigue item score from the MDASI-AML/MDS at weeks 8 and 12 for intensive and nonintensive chemotherapy patients, respectively.

The number and percentage of patients who experience AEs, SAEs, laboratory test abnormalities and clinically relevant ECG changes will be summarized.

Descriptive statistics (n, mean, standard deviation, coefficient of variation [CV], median, minimum, maximum and geometric mean, and its associated CV) will be used for PK parameters and PK concentrations.

Biomarker assessments will be summarized and analyzed using appropriate methods, such as Wilcoxon signedrank test. Correlations of biomarker results with PK parameters and measures of efficacy may be examined using Fisher's exact test, logistic regression, Kaplan-Meier analysis or linear regression, as appropriate. Treatment group comparison will be made using Wilcoxon rank-sum test, or linear mixed effects model, as appropriate.

Two interim analyses will be conducted after 50\% (futility only) and 70\% of deaths occur for the intensive chemotherapy trial. One interim analysis will be conducted after $60 \%$ of deaths occur for the nonintensive chemotherapy trial.

An external data monitoring committee (E-DMC) will be responsible for ongoing monitoring for patient safety in both blinded studies at least once yearly. The E-DMC will also evaluate the results of all interim analyses. The E-DMC will work with an unblinded reporting team separated from the blinded study team.

\section{Conclusion}

BRIGHT AML 1019 comprises two independent, separately powered Phase III, randomized, double-blind global studies designed to investigate whether glasdegib is superior to placebo in combination with cytarabine and daunorubicin (intensive trial) or azacitidine (nonintensive trial) in prolonging OS among patients with untreated AML. Upon completion, these studies will provide substantial evidence which may be useful to support expanding the current registration of glasdegib to include treatment of patients with AML in combination with ' $7+3$ ' or azacitidine, in addition to the current approved indication in combination with LDAC for patients aged 75 years or older or who have comorbidities that preclude use of intensive chemotherapy.

\section{Author contributions}

All authors met the International Committee of Medical Journal Editors' criteria for authorship, and were involved in the development and approval of the manuscript.

\section{Financial \& competing interests disclosure}

This study is sponsored by Pfizer. JE Cortes receives research support for his institution from BMS, Astellas, Arog, Daiichi, Pfizer, Novartis, Immunogen, Merus, Jazz, Takeda, BergenBio, Tolero, Sun Pharma, Trovagen and Amphivena. He receives consulting honorarium from Astellas, BMS, Daiichi, Pfizer, Novartis, Jazz and Biopath Holdings. H Dombret has a consulting or advisory role for Amgen, Astellas Pharma, AbbVie, Celgene, Cellectis, Daiichi Sankyo, Incyte, Janssen, Jazz Pharmaceuticals, Karyopharm Therapeutics, Immunogen, Shire-Baxalta, Menarini, Novartis, Otsuka, Pfizer, SERVIER and Sunesis Pharmaceuticals. He receives research funding from Amgen, Incyte, Novartis, Jazz Pharmaceuticals, SERVIER and Pfizer. AA Merchant acts as a consultant and on the advisory board for Agios and Pfizer, and receives research funding from Pfizer. T Tauchi is in the Speakers' Bureau for BristolMyers Squibb, Novartis and Pfizer. He receives research funding from Novartis. CG DiRienzo, B Sleight, XX Zhang, EP Leip, N Shaik, T Bell and G Chan are employees of Pfizer and own stock in Pfizer. MA Sekeres has a consulting or advisory role for Celgene and Millennium. The authors have no other relevant affiliations or financial involvement with any organization or entity with a financial interest in or financial conflict with the subject matter or materials discussed in the manuscript apart from those disclosed.

Medical writing support was provided by S Li of Engage Scientific Solutions and funded by Pfizer. 
Glasdegib in the treatment of acute myeloid leukemia

- Glasdegib is a smoothened inhibitor that has demonstrated potent and selective inhibition of Hedgehog (Hh) signaling in vitro, significant antitumor efficacy in vivo and clinical activity in Phase I and Ib/II trials in patients with hematologic malignancies, including untreated acute myeloid leukemia (AML) and high-risk myelodysplastic syndrome (MDS).

- Glasdegib plus standard of care chemotherapy was generally well tolerated, with a manageable safety profile; reported frequencies of alopecia, muscle spasms and dysgeusia appear lower than for other smoothened inhibitors.

The BRIGHT AML 1019 trial

- BRIGHT AML 1019 (NCT03416179, B1371019) includes two independent, separately powered Phase III, randomized (1:1), double-blind, global, placebo-controlled studies of chemotherapy in combination with glasdegib or placebo in adult patients with untreated AML.

- The intensive chemotherapy study consists of glasdegib $100 \mathrm{mg}$ once daily orally or placebo in combination with cytarabine and daunorubicin (' $7+3$ ') induction (second induction with ' $7+3$ ' or ' $5+2$ ' if needed), followed by consolidation with single-agent cytarabine, and/or hematopoietic stem cell transplantation (HSCT) for appropriate patients.

- The nonintensive chemotherapy study consists of glasdegib $100 \mathrm{mg}$ once daily orally or placebo plus azacitidine for at least 6 cycles. Treatment is continued in the absence of progressive disease or toxicity. HSCT may be performed as clinically indicated.

- For both studies, glasdegib/placebo therapy may be resumed postconsolidation or post-HSCT, but must be discontinued permanently upon documentation of confirmed measurable/minimal residual disease negativity postconsolidation or HSCT.

- For the intensive study participants, glasdegib/placebo may be continued for up to 2 years from randomization.

- Patients enrolled on the nonintensive study may continue glasdegib/placebo for as long as treatment is considered to confer clinical benefit.

Objectives and end points of the BRIGHT AML 1019 trial

- The primary objective is to investigate whether glasdegib is superior to placebo in combination with azacitidine or with ' $7+3$ ' chemotherapy in prolonging overall survival in patients with untreated AML.

- The primary end point is overall survival.

- The secondary objectives are:

- To compare other clinical efficacy measures, such as response rate and event-free survival.

- To estimate duration of response.

- To estimate time to response in the nonintensive trial.

- To compare fatigue scores, measured by MD Anderson Symptom Inventory AML/MDS Module (MDASI-AML/MDS) and other patient-reported outcomes measurements.

- To evaluate the overall safety profile.

- To characterize pharmacokinetics of glasdegib and its effects on QTC interval.

Conclusion

- BRIGHT AML 1019 comprises two independently powered Phase III, randomized, double-blind trials. On completion, the studies will provide substantial evidence that may be useful to support expanding the current registration of glasdegib to include the treatment of patients with AML in combination with ' $7+3$ ' chemotherapy regimen or with azacitidine.

\section{References}

Papers of special note have been highlighted as: $\bullet$ of interest; $\bullet \bullet$ of considerable interest

1. Global Burden of Disease Cancer Collaboration. Fitzmaurice C, Akinyemiju TF et al. Global, regional, and national cancer incidence, mortality, years of life lost, years lived with disability, and disability-adjusted life-years for 29 cancer groups, 1990 to 2016: a systematic analysis for the Global Burden of Disease study. JAMA Oncol. 4(11), 1553-1568 (2018).

2. Visser O, Trama A, Maynadie M et al. Incidence, survival and prevalence of myeloid malignancies in Europe. Eur. J. Cancer 48(17), 3257-3266 (2012).

3. American Cancer Society. Cancer facts and figures. American Cancer Society 2019. Atlanta, GA. www.cancer.org/content/dam/cancerorg/research/cancer-facts-and-statistics/annual-cancer-facts-and-figures/2019/cancer-facts-and-figures-2019.pdf

4. Wiese M, Daver N. Unmet clinical needs and economic burden of disease in the treatment landscape of acute myeloid leukemia. Am. J. Manag. Care 24(Suppl. 16), S347-S355 (2018).

5. Lyle L, Daver N. Current and emerging therapies for patients with acute myeloid leukemia: a focus on MCL-1 and the CDK9 pathway. Am. J. Manag. Care 24(Suppl. 16), S356-S365 (2018).

6. Short NJ, Rytting ME, Cortes JE. Acute myeloid leukaemia. Lancet 392(10147), 593-606 (2018). 
7. Wang X, Huang S, Chen JL. Understanding of leukemic stem cells and their clinical implications. Mol. Cancer 16(1), 2 (2017).

8. Thomas D, Majeti R. Biology and relevance of human acute myeloid leukemia stem cells. Blood 129(12), 1577-1585 (2017).

9. Pollyea DA, Jordan CT. Therapeutic targeting of acute myeloid leukemia stem cells. Blood 129(12), 1627-1635 (2017).

10. Thomas BJ. Cell-cycle control during development: taking it up a notch. Dev. Cell 8(4), 451-452 (2005).

11. Queiroz KC, Ruela-De-Sousa RR, Fuhler GM et al. Hedgehog signaling maintains chemoresistance in myeloid leukemic cells. Oncogene 29(48), 6314-6322 (2010).

12. Lin TL, Matsui W. Hedgehog pathway as a drug target: smoothened inhibitors in development. Onco. Targets Ther. 5, 47-58 (2012).

-• Aberrant Hedgehog pathway signaling has been found in various solid and hematologic malignancies. Antagonists of the signal transducer smoothened showed encouraging anticancer results in preclinical studies and in Phase I and II clinical trials.

13. Khan AA, Harrison CN, Mclornan DP. Targeting of the Hedgehog pathway in myeloid malignancies: still a worthy chase? $B r$. J. Haematol. 170(3), 323-335 (2015).

14. Aberger F, Hutterer E, Sternberg C, Del Burgo PJ, Hartmann TN. Acute myeloid leukemia - strategies and challenges for targeting oncogenic Hedgehog/GLI signaling. Cell Commun. Signal. 15(1), 8 (2017).

- Agents targeting the Hedgehog/glioma-associated oncogene homolog signaling pathway in combination with other agents targeting important mechanisms involved in acute myeloid leukemia (AML) pathology may provide new treatment options.

15. Ingham PW, Mcmahon AP. Hedgehog signaling in animal development: paradigms and principles. Genes Dev. 15(23), 3059-3087 (2001).

16. Sadarangani A, Pineda G, Lennon KM et al. GLI2 inhibition abrogates human leukemia stem cell dormancy. J. Transl. Med. 13, 98 (2015).

17. Fukushima N, Minami Y, Kakiuchi S et al. Small-molecule Hedgehog inhibitor attenuates the leukemia-initiation potential of acute myeloid leukemia cells. Cancer Sci. 107(10), 1422-1429 (2016).

18. Tauchi T, Okabe S, Katagiri S, Tanaka Y, Tohyama K, Ohyashiki K. Targeting the Hedgehog signaling pathway by glasdegib limits the self-renewal of MDS-derived induced potent stem cells (iPSC). J. Cancer Sci. Ther. 9(6), 479-484 (2017).

19. Kakiuchi S, Minami Y, Miyata Y et al. NANOG expression as a responsive biomarker during treatment with hedgehog signal inhibitor in acute myeloid leukemia. Int. J. Mol. Sci. 18(3), 486 (2017).

20. Tauchi T, Okabe S, Katagiri S et al. Combining effects of the SMO inhibitor and BCL-2 inhibitor in MDS-derived induced potent stem cells (iPSC). Blood 130(Suppl. 1), 1249 (2017).

21. Chaudhry P, Singh M, Triche TJ, Guzman M, Merchant AA. GLI3 repressor determines Hedgehog pathway activation and is required for response to SMO antagonist glasdegib in AML. Blood 129(26), 3465-3475 (2017)

22. Martinelli G, Oehler VG, Papayannidis C et al. Treatment with PF-04449913, an oral smoothened antagonist, in patients with myeloid malignancies: A Phase I safety and pharmacokinetics study. Lancet Haematol. 2(8), 00096-00094 (2015).

- The maximum tolerated dose of glasdegib was $400 \mathrm{mg}$ once daily. The most common treatment-related adverse events included dysgeusia, decreased appetite and alopecia. Pharmacokinetics were dose proportional. The recommended Phase II dose was $200 \mathrm{mg}$ or lower once daily.

23. Borate U, Smith BD, Gore $\mathrm{S}$ et al. Phase IB study of glasdegib (PF-04449913) in combination with azacitidine in patients with higher risk myelodysplastic syndrome, oligoblastic acute myeloid leukemia, or chronic myelomonocytic leukemia. Haematologica 101(Suppl. 1), 73-74 (2016).

- Glasdegib plus azacitidine showed evidence of clinical activity with no evidence of drug-drug interaction; complete remissions appeared favorable in the context of the $15-17 \%$ complete remission rate seen with azacitidine alone.

24. Minami Y, Minami H, Miyamoto T et al. Phase I study of glasdegib (PF-04449913), an oral smoothened inhibitor, in Japanese patients with select hematologic malignancies. Cancer Sci. 108(8), 1628-1633 (2017).

- Glasdegib (100 mg once-daily oral dose) was safe and well tolerated in Japanese patients.

25. Savona M, Pollyea D, Stock W et al. Phase Ib study of glasdegib, a Hedgehog pathway inhibitor, in combination with standard chemotherapy in patients with AML or high-risk MDS. Clin. Cancer Res. 24(10), 2294-2303 (2018).

-• NCT01546038 evaluated the safety, pharmacokinetics, pharmacodynamics and clinical activity of glasdegib (PF-04449913), a novel Hedgehog pathway smoothened inhibitor, in patients with AML or high-risk myelodysplastic syndromes, showing that treatment with glasdegib in combination with standard chemotherapy was generally well tolerated and consistent with prior findings.

26. Cortes JE, Douglas Smith B, Wang ES et al. Glasdegib in combination with cytarabine and daunorubicin in patients with AML or high-risk MDS: Phase II study results. Am. J. Hematol. 93(11), 1301-1310 (2018).

-• Glasdegib plus cytarabine/daunorubicin was well tolerated and associated with clinical activity (i.e., low rate of relapse and a suggestion of a favorable overall survival) in patients with untreated AML or high-risk myelodysplastic syndromes.

27. Cortes JE, Heidel FH, Hellmann A et al. Randomized comparison of low dose cytarabine with or without glasdegib in patients with newly diagnosed acute myeloid leukemia or high-risk myelodysplastic syndrome. Leukemia 33(2), 379-389 (2019). 
-• Glasdegib plus low-dose cytarabine resulted in a favorable benefit-to-risk profile (i.e., significant and clinically meaningful improvement in overall survival compared with the standard therapy of low-dose cytarabine and generally manageable toxicity) and may be a promising option for AML patients unsuitable for intensive chemotherapy.

28. Gerds AT, Tauchi T, Ritchie E et al. Phase I/II trial of glasdegib in patients with primary or secondary myelofibrosis previously treated with ruxolitinib. Leuk. Res. 79, 38-44 (2019).

29. Wagner AJ, Messersmith WA, Shaik MN et al. A Phase I study of PF-04449913, an oral hedgehog inhibitor, in patients with advanced solid tumors. Clin. Cancer Res. 21(5), 1044-1051 (2015).

30. Larson RA. Induction therapy for acute myeloid leukemia in younger adults. UpToDate. UpToDate Inc, Waltham, MA, USA. http://www.uptodate.com

31. Estey E. Acute myeloid leukemia and myelodysplastic syndromes in older patients. J. Clin. Oncol. 25(14), 1908-1915 (2007).

32. Döhner H, Estey E, Grimwade D et al. Diagnosis and management of AML in adults: 2017 ELN recommendations from an international expert panel. Blood 129(4), 424-447 (2017)

33. Löwenberg B. Sense and nonsense of high-dose cytarabine for acute myeloid leukemia. Blood 121(1), 26-28 (2013).

34. Schuh AC, Döhner H, Pleyer L, Seymour JF, Fenaux P, Dombret H. Azacitidine in adult patients with acute myeloid leukemia. Crit. Rev. Oncol. Hematol. 116, 159-177 (2017).

35. Dombret H, Seymour JF, Butrym A et al. International Phase III study of azacitidine vs conventional care regimens in older patients with newly diagnosed AML with >30\% blasts. Blood 126(3), 291-299 (2015).

36. Tibes R, Al-Kali A, Oliver GR et al. The Hedgehog pathway as targetable vulnerability with 5-azacytidine in myelodysplastic syndrome and acute myeloid leukemia. J. Hematol. Oncol. 8, 114 (2015).

37. Röllig C, Bornhäuser M, Thiede $\mathrm{C}$ et al. Long-term prognosis of acute myeloid leukemia according to the new genetic risk classification of the European LeukemiaNet recommendations: evaluation of the proposed reporting system. J. Clin. Oncol. 29(20), 2758-2765 (2011).

38. Arber DA, Orazi A, Hasserjian R et al. The 2016 revision to the World Health Organization classification of myeloid neoplasms and acute leukemia. Blood 127(20), 2391-2405 (2016).

39. Fernandez HF, Sun Z, Yao X et al. Anthracycline dose intensification in acute myeloid leukemia. N. Engl. J. Med. 361(13), 1249-1259 (2009).

40. Löwenberg B, Ossenkoppele GJ, Van Putten W et al. High-dose daunorubicin in older patients with acute myeloid leukemia. N. Engl. J. Med. 361(13), 1235-1248 (2009).

41. Przepiorka D, Weisdorf D, Martin P et al. 1994 Consensus Conference on acute GvHD grading. Bone Marrow Transplant. 15(6), $825-828$ (1995).

42. Jagasia MH, Greinix HT, Arora $\mathrm{M}$ et al. National Institutes of Health consensus development project on criteria for clinical trials in chronic graft-versus-host disease: I. The 2014 diagnosis and staging working group report. Biol. Blood Marrow Transplant. 21(3), 389.e381-401.e381 (2015).

43. Shulman HM, Cardona DM, Greenson JK et al. NIH Consensus development project on criteria for clinical trials in chronic graft-versus-host disease: II. The 2014 pathology working group report. Biol. Blood Marrow Transplant. 21(4), 589-603 (2015).

44. Kalbfleisch JD, Prentice RL. The Statistical Analysis of Failure Time Data. John Wiley \& Sons, Inc, New York, USA (1980).

45. Brookmeyer R, Crowley J. A confidence interval for the median survival time. Biometrics 38(1), 29-41 (1982). 
